\title{
Seismic Hazard Analysis of Low Seismic Regions, Durg \& Rajnandgaon
}

\author{
Ashish Kumar Parashar ${ }^{1}$, Dr. Sohanlal Atmapoojya ${ }^{2}$, Dr. S.S. Rathore ${ }^{3}$ \\ ${ }^{I}$ (Department of Civil Engineering, Institute of Technology, GGV (Central University) Bilaspur, C.G.,India \\ ${ }_{2}^{2}$ (Department of Civil Engineering, K.I.T.S. Ramtek, Maharashtra, India) \\ ${ }^{3}$ (Principal, M.I.E.T. Kudwa, Gondia, Maharashtra, India)
}

\begin{abstract}
Seismic hazard assessment of low seismicity regions of the world is now-a-days becoming more common. The seismic hazard assessment involves the quantitative estimation of ground motion characteristics at a particular site. Seismic Hazard is a regional property. It can neither be prevented nor reduced. The only alternative is to quantify the Hazard and minimize the possible damages to the structures due to possible strong Ground Motion. Durg and Rajnandgaon sites are two District Headquarters of the state of Chhattisgarh. In the present study. Deterministic Seismic Hazard Assessment (DSHA) has been applied to these District Headquarters to assess the maximum Peak Ground Acceleration (PGA) at these sites. Beauro of Indian Standard has specified these sites in seismic Zone II. This fact has been verified in the present study.
\end{abstract}

Keywords - Deterministic Seismic Hazard, Fault Map, Earthquake, Peak Ground Acceleration, District Headquarters

\section{INTRODUCTION}

A spate of Earthquakes in recent past, causing extensive damage has heightened the sensitivity of Engineers and Planners to the looming seismic risk in densely populated cities, major dams and important \& historical places. Earthquakes are a global phenomenon and a global problem. It is not possible to prevent Earthquakes from occurring but it is possible to mitigate the effects of strong Earthquake shaking, to reduce loss of lives, injuries and damages. Occurrence of one or more Earthquakes at a project site is known as Seismic Hazard.

The Earthquakes in India occur in the plate boundary of the Himalayas region as well as in the intraplate region of peninsular India (P I). Devastating events have occurred in P I in the recent past, which must be considered as a severe warning about the possibility of such Earthquake in the future. Engineering approaches to Earthquake resistant design will be successful to the extent that the forces due to future shocks are accurately estimated at location of a given structure. Earthquakes are low probability events, but with very high levels of risks to the society. Hence, either under estimation or over estimation of seismic hazard will prove dangerous or costly in the end Earthquakes present a threat to people and the facilities they design and build. Seismic hazard analysis (SHA) is the evaluation of potentially damaging earthquake related phenomenon to which a facility may be subjected during its useful lifetime. Seismic hazard analysis is done for some practical purpose, typically seismic-resistant design or retrofitting.

Seismic hazards may be analyzed deterministically as and when a particular earthquake scenario is assumed, or probabilistically, in which uncertainties in earthquake size, location, and time of occurrence are explicitly considered (Kramer 1996). In practice, DSHAs often assume that earthquakes of the largest possible magnitude occur at the shortest possible distance to the site within each source zone. The earthquake that produces the most severe site motion is then used to compute site -specific ground motion parameters. Deterministic method is the technique in which a single estimate of parameters is used to perform each analysis. To account for uncertainty, several analyses may be conducted with different parameters. For assessment of PGA, of District Headquarters Durg and Rajnandgaon have been considered for this study. The present study details of these District Headquarters sites are as follows:

\begin{tabular}{|l|c|c|}
\hline \multicolumn{1}{|c|}{ Salient Features } & Durg & Rajnandgaon \\
\hline Latitude & $21^{\circ} 11^{\prime} \mathrm{N}$ & $21^{\circ} 05^{\prime} \mathrm{N}$ \\
\hline Longitude & $81^{\circ} 05^{\prime} \mathrm{E}$ & $81^{\circ} 05^{\prime} \mathrm{E}$ \\
\hline
\end{tabular}




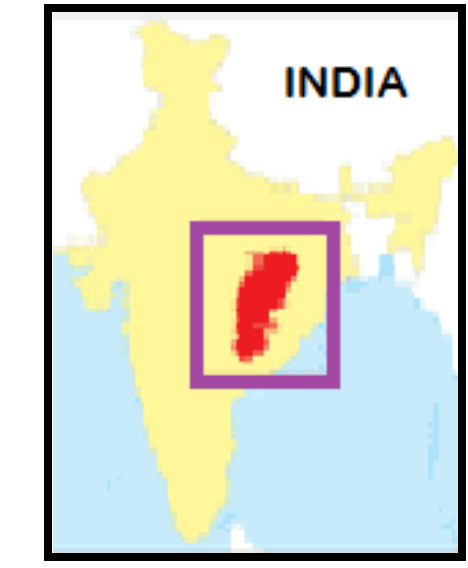

Figure1.1 (a) Chhattisgarh State

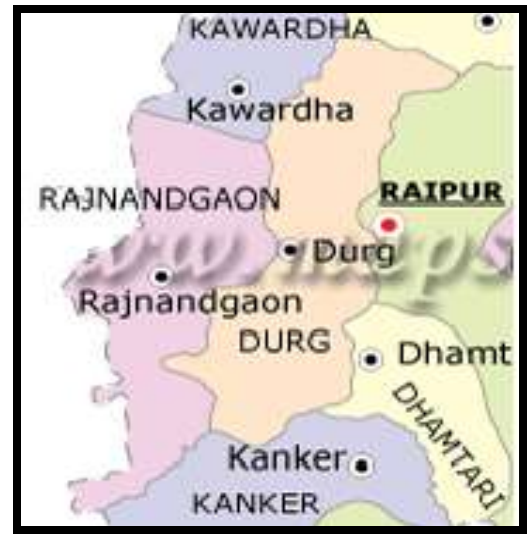

Figure1.1 (b) District Headquarters Durg and Rajnandgaon

To evaluate the seismic hazards for a particular site or region, all possible sources of seismic activity must be identified and their potential for generating future strong ground motion needs to be evaluated. Identification of seismic sources requires some detective work, nature's clues, some of which are obvious and others quite obscure, must be observed and interpreted.

Seismic hazard analysis involves the quantitative estimation of ground shaking hazards at a particular area. The most important factors affecting seismic hazard at a location are:

1. Earthquake magnitude

2. The source-to-site Distance

3. Earthquake rate of Occurrence (return period)

4. Duration of Ground Shaking

\section{DETERMINISTIC SEISMIC HAZARD ASSESSMENT (DSHA)}

The DSHA can estimate in the following steps:

- Seismic Sources.

- $\quad$ Earthquake- recurrence- frequency.

- Deaggregation of Seismic Hazard.

- $\quad$ Ground motion attenuation.

- $\quad$ Estimation of PGA

\subsection{Seismic Sources}

- A circular region of $300 \mathrm{~km}$ radius has to be assumed around the site.

- $\quad$ Seismicity information has to be collected (i.e Epicenter, Magnitude) inside the $300 \mathrm{~km}$ radius.

- Different faults in this $300 \mathrm{Km}$. radius region have to be identified, length of the fault and their shortest distances from the site have to be worked out.

\subsubsection{Earthquake History of Study Area}

Most of the earthquakes occurred in India are in northern part of Indian sub continent. These occurred due to the upward movement of Himalayan region. The earthquake data prior to 1827 is not available. The available data is from 1846- 2011. However, due to non availability of earthquake data United States Geological survey was collected with radial search of $300 \mathrm{Km}$. The collected earthquake data is as shown in Appendix I, for Durg and Rajnandgaon district headquarters. As per the past records the earthquake magnitudes Mw of 3 to 6.7 are available. A historical record of past Earthquakes, in a region, is the one of the most important tool as these records are useful to assess the region seismicity.

\subsection{Earthquake Recurrence Frequency}

Earthquake Recurrence relationship has to be worked in the following steps:

- $\quad$ Earthquake information for region has to be collected over a long period from various historical records.

- $\quad$ All the data has to be arranged as per the number of Earthquakes that exceeded various magnitude values $(\mathrm{m}=0,1,2,3$,

- $\quad$ Suitable Earthquake Recurrence Relation has to be used, which appropriately characterizes the seismicity of the region.

\subsection{Deaggregation of Seismic Hazard}


1. In DSHA, the basic idea is to foreshadow on each of the causative fault, the magnitude of an Earthquake, which may be exceeded in say 100 years or 1000 years.

2. M100 has to be worked out for each fault.

3. Using the Regional Recurrence Relation, it is easy to find the above magnitudes for the region, but not for individual faults.

4. The potential of a fault to produce an Earthquake of a particular magnitude would depend on the length of the fault itself.

5. $\mathrm{Ni}(\mathrm{m} 0)$ on any individual fault may be proportional to the length of the fault itself.

Weightage $\mathrm{Wi}=\mathrm{Li} / \Sigma \mathrm{Li}$.

6. The ' $b$ ' value of any fault is to be same as the regional ' $b$ ' value.

7. The value of mmax for each fault is to be fixed up by finding the most probable magnitude of the largest past event that can be associated with the fault. This value is increased by 0.5 and taken as $\mathrm{m}_{\max }$. In case, only the highest intensity value is known, the event magnitude is taken as $m=2 / 3($ I0 $)+1$.

\subsection{Ground Motion Attenuation}

- Attenuation may be described as the way in which strong motion parameters decay with distance from the source.

- This depends on the source properties (M, focal depth, fault type and size), as well as on the regional properties (frequency dependent damping, layering, anisotropy etc.).

- The property of the site (hard rock, soft soil, valley and mountain) also influences the ground motion attenuation.

For the present study attenuation relationship5 suggested by R N Iyengar \& S T G Raghukant, (Applicable for peninsular India, under bed rock condition) has been used.

In $(\mathrm{PGA} / \mathrm{g})=\mathrm{C} 1+\mathrm{C} 2(\mathrm{~m}-6)+\mathrm{C} 3(\mathrm{~m}-6) 2-\ln (\mathrm{R})-\mathrm{C} 4(\mathrm{R})+\ln \varepsilon$

Where,

$\mathrm{C} 1=1.6858, \mathrm{C} 2=0.9241, \mathrm{C} 3=0.0760, \mathrm{C} 4=0.0057$,

$\mathrm{R}=$ Hypo central distance, $\mathrm{m}=$ magnitude, $\ln \varepsilon=0$ (for DSHA).

\subsection{Estimation of Peak Ground Acceleration (PGA)}

The PGA, which exceedes with $50 \%$ probability, is to be calculated from the attenuation equation. In DSHA, the maximum among these values is to be taken as the design basis acceleration depending on the acceptability of this value based on other seismological considerations. This PGA value could be a reference value for further work.

\section{APPLICATION OF DSHA}

Deterministic seismic hazard analysis (DSHA) has been applied to Durg and Rajnandgaon sites using the following steps: A region of $300 \mathrm{~km}$ radius around both Durg and Rajnandgaon sites were considered and all the faults having $\geq 25 \mathrm{~km}$ length were marked. These regions are shown in Figure 3.1 and Figure 3.2 respectively

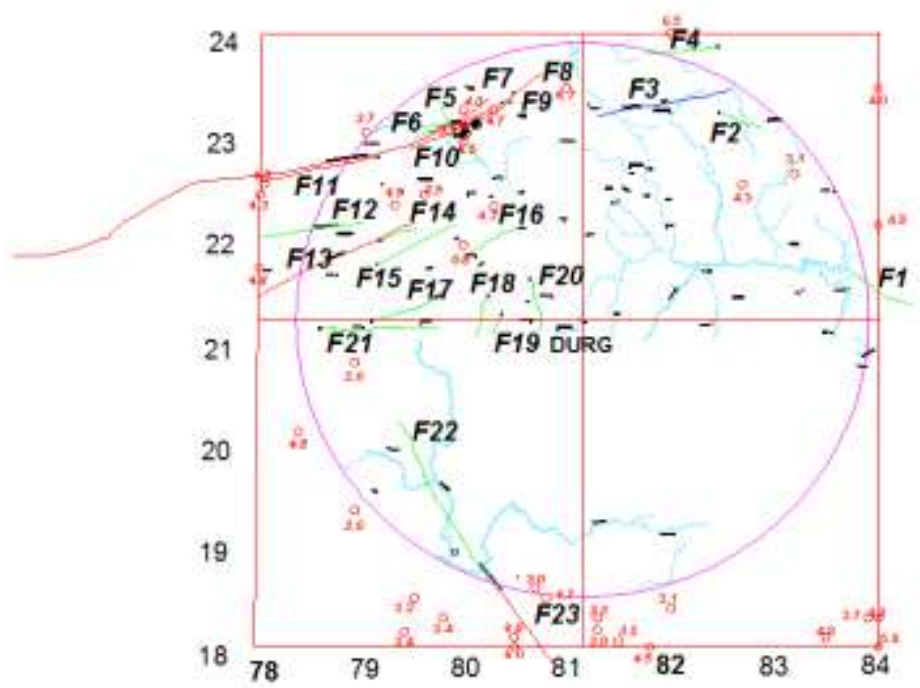

Figure 3.1 Fault considered for Deterministic Seismic Hazard Analysis of District Headquarter Durg 


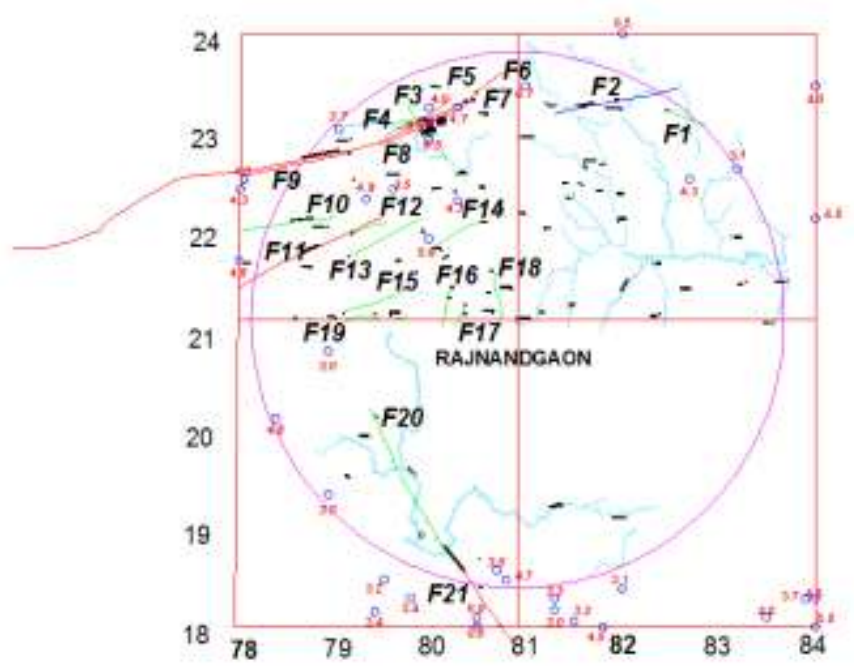

Figure 3.2 Fault considered for Deterministic Seismic Hazard Analysis of District Headquarter Rajnandgaon

With the help of different literature available and websites 55 Nos. of Earthquakes in the magnitude range $3 \geq \mathrm{Mw}$ to $\mathrm{Mw} \leq 6.7$ for Durg and Rajnandgaon sites over the period from 1846 to 2012 (166) years have been collected. The same is presented at Appendix I.

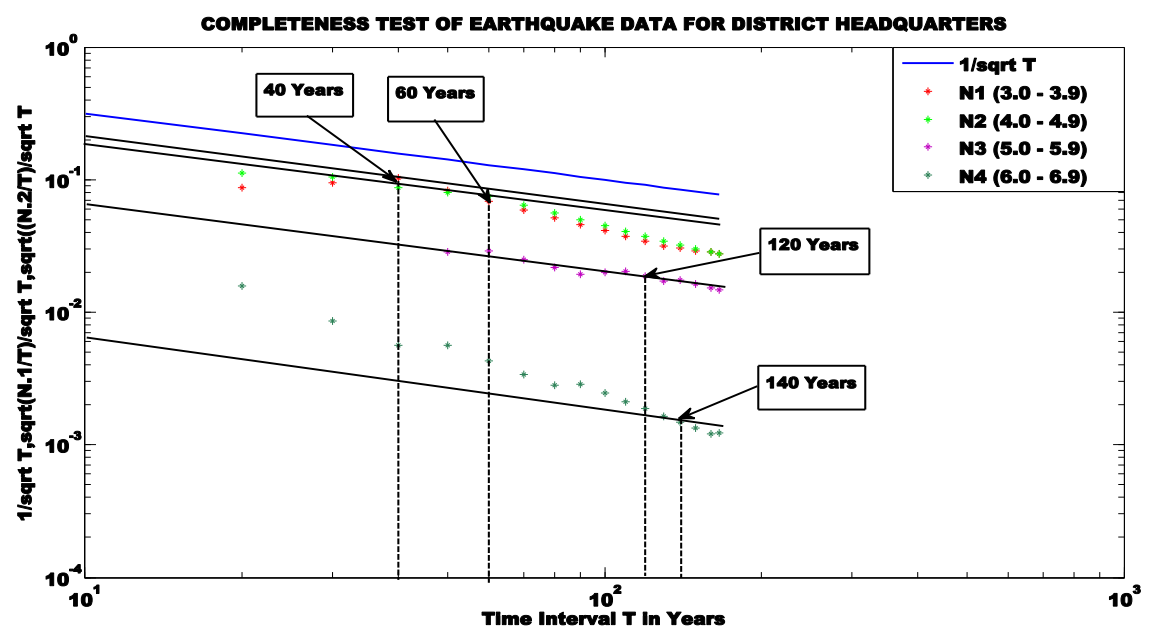

Figure 3.3 Completeness Test of Earthquake Data for District Headquarters

The completeness analysis for District Headquarters sites has been performed and as shown in Figure 3.3. Earthquakes data for completeness test for Durg and Rajnandgaon sites have been presented in Table 2.3 (Appendix II) respectively. Completeness test of Earthquakes data for Durg and Rajnandgaon sites has been shown in Figure 3.3. It has been observed for Durg and Rajnandgaon (from the Table 3.1 below) that 3.0 magnitude will be completed in 40 years time interval while 6.7 magnitude will complete in 140 .

Table No.3.1 Activity Rate and Interval of Completeness at District Headquarters

\begin{tabular}{|c|c|c|c|}
\hline Magnitude Mw & No. of Events $\geq \mathrm{Mw}$ & Complete in interval & No. of Events per year \\
\hline 3.0 & 55 & 40 & 1.3750 \\
\hline 4.0 & 34 & 60 & 0.5667 \\
\hline 5.0 & 13 & 120 & 0.1084 \\
\hline 6.7 & 3 & 140 & 0.0214 \\
\hline
\end{tabular}

Using completeness analysis, Regional Recurrence Relationship has been obtained for: District Headquarters

$$
\log 10(\mathrm{~N})=1.99-0.5478 \mathrm{Mw}-
$$




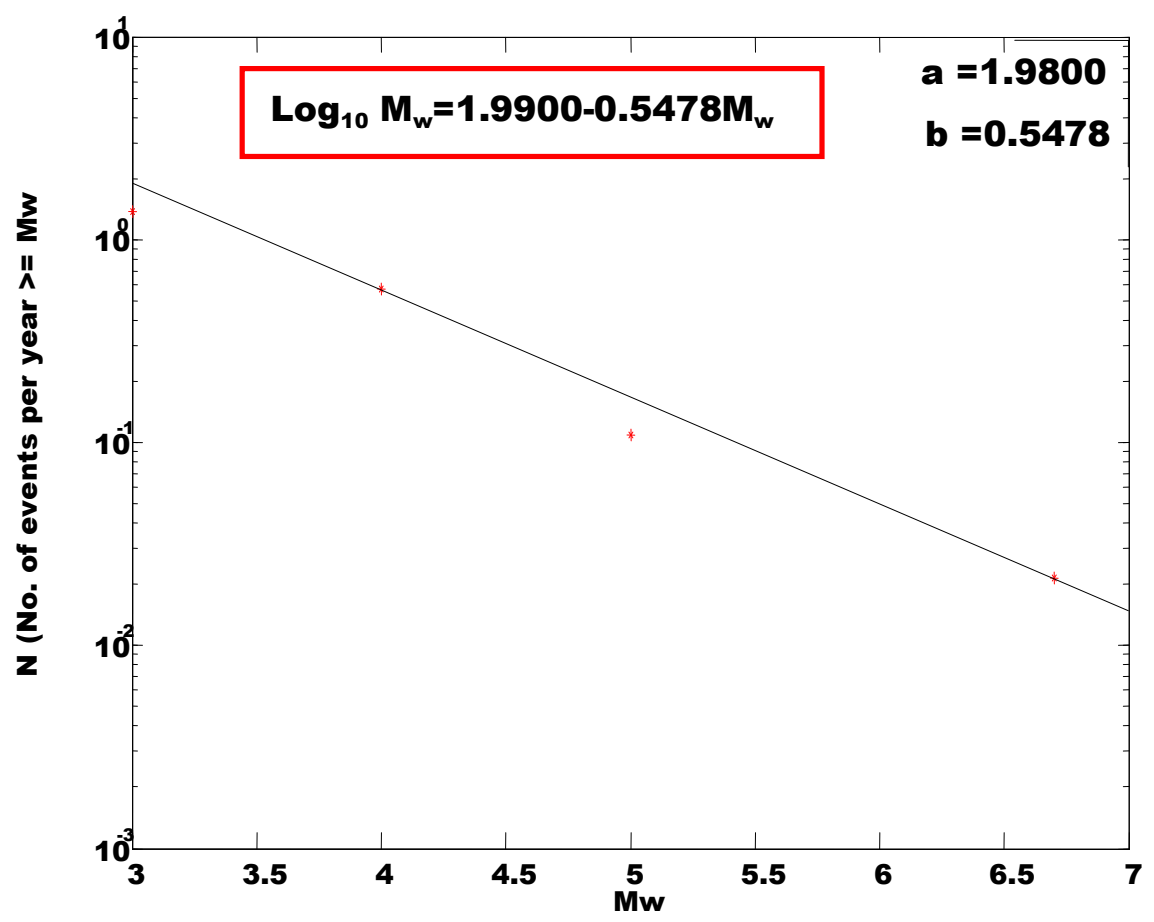

Figure 3.4 Regional Recurrence Relationships for District Headquarters

The Hypo-central distance (by considering the focal depth as $10 \mathrm{~km}$ ), weightage and maximum potential magnitude $(\mathrm{Mu})$ is obtained for each fault having length $\geq 25 \mathrm{~km}$ and has been presented in Table 2.4 for Durg and in Table 2.5 (Appendix II) for Rajnandgaon.

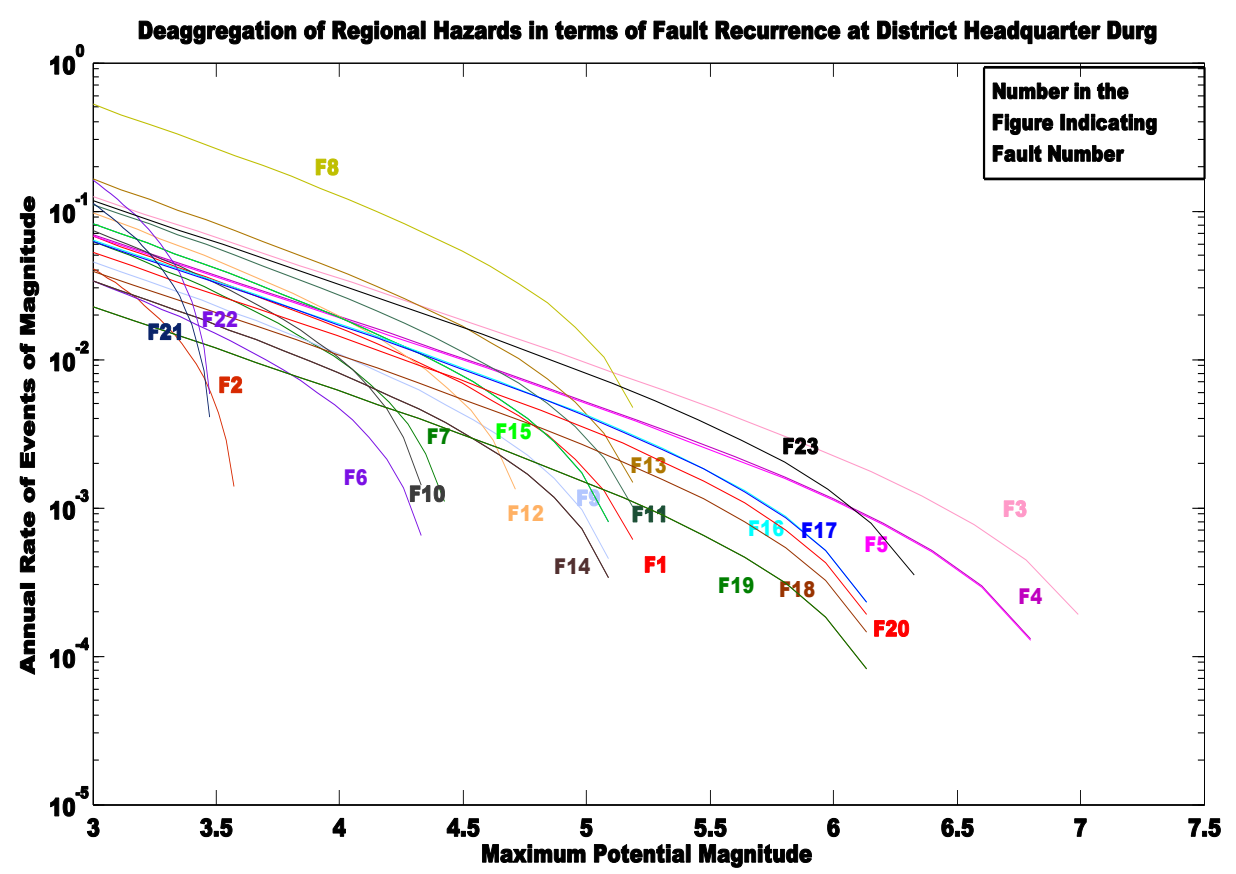

Figure 3.5 Deaggregation of Regional Hazards in terms of Fault Recurrence at District Headquarter Durg 


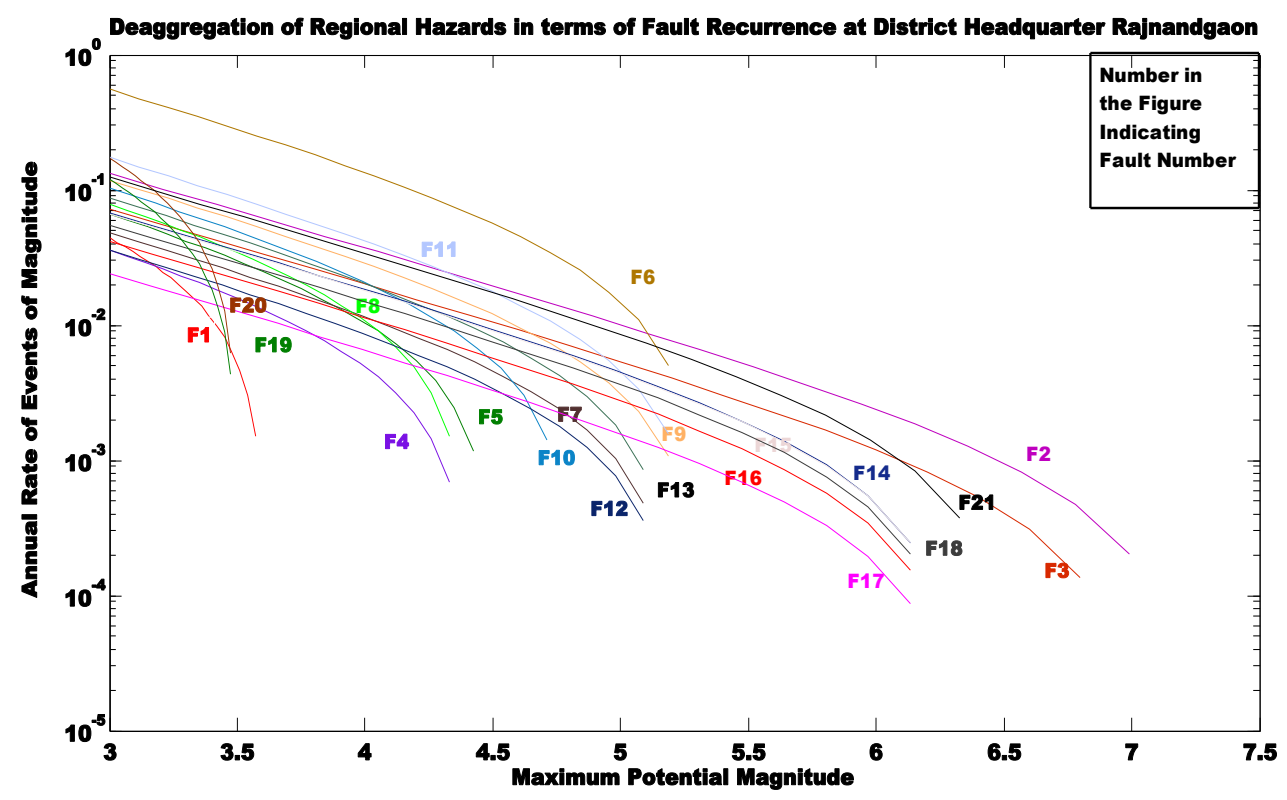

Figure 3.6 Deaggregaton of regional hazards in terms of fault recurrence at District Headquarter Rajnandgaon M100 has been obtained by generating the fault deaggregation record. In this study all the faults having $\geq 25 \mathrm{~km}$ lengths are considered. Fault deaggregation for Durg and Rajnandgaon have been shown in Figure 3.5 and Figure 3.6 respectively.

\section{RESULT \& CONCLUSION}

Regional Recurrence Relationship obtained for Durg and Rajnandgaon sites have been presented in Equation No 3.1 Obtained "b" value 0.5478 respectively. Hence, the both the sites are situated in less seismic active zone. Deterministic Seismic Hazard Analysis has been applied to the District Headquarters Durg and Rajnandgaon sites, Values of P.G.A. for M100 Earthquakes have been presented in Table No.2.6 \& Table No.2.7 ( Appendix II) respectively. Maximum values of Peak Ground Acceleration (P.G.A.) for Durg Site has been obtained due to fault No. 20 (length $58 \mathrm{~km}$, Distance $48.093 \mathrm{~km}$ ) is equal to $0.01385 \mathrm{~g}$. and Maximum value of Peak Ground Acceleration (P. G. A.) for Rajnandgaon. Site has been obtained due to fault No. 18 (length $58 \mathrm{~km}$, Distance $21.245 \mathrm{~km}$ ) is equal to 0.03433g. As per IS 1893:2002(Part-1) the District Headquarters region have been categorized as zone II and corresponding P.G.A. is equal to $0.1 \mathrm{~g}$. Hence, this fact has also been verified from the present study.

\section{REFERENCES}

[1] Attenuation of Strong Ground Motion in Peninsular India. R N Iyenger and S T G Raghukant. Seismological Research Letters. Volume 75, Number 4, July/August 2004, pp530-539.

[2] Criteria for Earthquake Resistant Design of Structures (Part, General Provisions and Buildings, IS-1893:2002.

[3] Cornell, C.A., Engineering Seismic Risk Analysis. B. Seismological Society of America, 58, 1968, pp 1583-1606

[4] Catalogue of Earthquakes in India and Neighborhood, (From Historical period up to 1979) Indian Society of Earthquak Technology, Roorkee-1993

[5] Guttenberg, B and Richter, C.F., Frequency of earthquakes in California. B. Seismological Society of America, 34, 1944, pp 185188.

[6] Krammer, S.L., Geotechnical Earthquake Engineering, Pearson Education Ptd. Ltd. Reprinted 2003, Delhi, India, 1996.

[7] Microzonation of Earthquake Hazard in Greater Delhi Area. R N Iyenger and S Ghose. Current Science. Vol.87, No. 9, 10,November 2004, pp 1193-1201.

[8] Rao, R. B. and Rao, S. P. (1984): 'Historical Seismicity of Peninsular India', Bulletin of the Seismological Society ofAmerica, 74, pp. 2519-2533.

[9] Raghu Kanth, S. T. G. and Iyengar, R. N. (2007): 'Estimation of Seismic Spectral Acceleration in Peninsular India', Journal of Earth System Science, 116, pp. 199-214.

[10] Steven L. Kramer. Geotechnical Earthquake Engineering, Pearson Education (Singapore) Ltd. 1996.

[11] Seismic Tectonic Atlas of India and its Environs Geological Survey of India 2000.

[12] U. S. Geological Survey Earthquake Database (Website). 
Seismic Hazard Analysis Of Low Seismic Regions, Durg \& Rajnandgaon APPENDIX 1

Listing of Earthquake Events around District Head Quarters

\begin{tabular}{|c|l|c|c|c|c|c|c|}
\hline \multirow{2}{*}{ S.No. } & \multirow{2}{*}{$\begin{array}{l}\text { Name of Head } \\
\text { Quarter }\end{array}$} & \multicolumn{2}{|c|}{ Location of HQ } & \multicolumn{2}{|c|}{ Latitude } & \multicolumn{2}{c|}{ Longitude } \\
\cline { 3 - 8 } & & Latitude & Longitude & \multicolumn{2}{|c|}{} \\
\hline 1 & Durg & $21^{\circ} 11^{\prime} \mathrm{N}$ & $81^{\circ} 21^{\prime} \mathrm{E}$ & $18^{\circ} 00^{\prime}$ & $24^{\circ} 0^{\prime}$ & $78^{\circ} 00^{\prime}$ & $84^{\circ} 0^{\prime}$ \\
\hline 2 & Rajnandgaon & $21^{\circ} 05^{\prime} \mathrm{N}$ & $81^{\circ} 05^{\prime} \mathrm{E}$ & $18^{\circ} 0^{\prime}$ & $24^{\circ} 0^{\prime}$ & $78^{\circ} 00^{\prime}$ & $84^{\circ} 0^{\prime}$ \\
\hline
\end{tabular}

\begin{tabular}{|c|c|c|c|c|c|c|c|c|c|c|c|}
\hline S No. & Year & Month & Date & Latitude & Longitude & Int & Ms & $\mathrm{Mb}$ & $\mathrm{Mw}$ & Depth & Source \\
\hline 1 & 1846 & 5 & 27 & 23 & 80 & $\mathrm{Vi}$ & & & 6.5 & & OLD, NEIC, UKOLD \\
\hline 2 & 1858 & 10 & 12 & 18.3 & 84 & $\mathrm{~V}$ & & & 4.3 & & OLD, NEIC \\
\hline 3 & 1859 & 8 & 24 & 18.1 & 83.5 & $\mathrm{~V}$ & & & 3.7 & & OLD, NEIC, UKGSI \\
\hline 4 & 1861 & 11 & 13 & 18.11 & 83.5 & Iii & & & 3 & & UGS \\
\hline 5 & 1871 & 9 & 27 & 18.3 & 83.9 & Iii & & & 3 & & UGS \\
\hline 6 & 1872 & 11 & 22 & 18.86 & 80 & $\mathrm{Vi}$ & & & 5 & & UGS \\
\hline 7 & 1878 & 12 & 10 & 18.3 & 83.9 & $\mathrm{Iv}$ & & & 3.7 & & UGS \\
\hline 8 & 1903 & 5 & 17 & 23 & 80 & & 5 & & 5.5 & & TRI, NEIC, UKTRI \\
\hline 9 & 1917 & 4 & 17 & 18 & 84 & & 5.5 & & 5.8 & & ISS, NEIC, UKIMD \\
\hline 10 & 1927 & 6 & 2 & 23.5 & 81 & & 6.5 & 6 & 6.7 & & GR \\
\hline 11 & 1927 & 6 & 2 & 24 & 82 & & & & 6.5 & & NEIC, UKCH \\
\hline 12 & 1954 & 1 & 5 & 18 & 81.8 & & 4 & & 4.5 & & IMD \\
\hline 13 & 1954 & 1 & 5 & 18 & 81.3 & & & & 4 & & NEIC, UKIMD \\
\hline 14 & 1957 & 8 & 25 & 22 & 80 & & 5.5 & 5.5 & 5.8 & & SHL, NEIC, UKSHL \\
\hline 15 & 1959 & 8 & 9 & 18.1 & 83.5 & & 4.1 & & 4.7 & & RAO, UKRAO \\
\hline 16 & 1959 & 12 & 23 & 18.1 & 83.5 & & 4.3 & & 4.8 & & RAO, UKRAO \\
\hline 17 & 1965 & 4 & 29 & 23.5 & 84 & & & & 4 & & NEIC \\
\hline 18 & 1968 & 11 & 14 & 21.8 & 78 & & 4.2 & & 4.8 & & IMD, NEIC, UKHYB \\
\hline 19 & 1969 & 4 & 14 & 18 & 80.5 & & & 5.2 & 5.3 & & IMD \\
\hline 20 & 1969 & 4 & 15 & 18 & 80.7 & & & 4.6 & 4.6 & 33 & ISC \\
\hline 21 & 1969 & 4 & 14 & 18.1 & 80.5 & & & & 6 & & UKTS \\
\hline 22 & 1969 & 3 & 26 & 22.6 & 78.1 & & 4.2 & & 4.8 & & IMD \\
\hline 23 & 1969 & 4 & 14 & 18 & 80.5 & & & & 6 & & USC \\
\hline 24 & 1969 & 4 & 14 & 18 & 80.5 & $\mathrm{Vi}$ & & & 5.7 & 33 & USC \\
\hline 25 & 1973 & 7 & 12 & 23.2 & 80 & & 4 & & 4.6 & & IMD \\
\hline 26 & 1973 & 7 & 12 & 23.1 & 79 & & & & 3.7 & & NEIC, UKHYB \\
\hline 27 & 1975 & 4 & 24 & 18.7 & 80.7 & & 3 & & 3 & & INR, NEIC, UKHYB \\
\hline 28 & 1975 & 7 & 3 & 18 & 79.5 & & 3.2 & & 3.2 & & INR \\
\hline 29 & 1975 & 9 & 15 & 18.4 & 79.2 & & 3.2 & & 3.2 & & INR, NEIC, UKHYB \\
\hline 30 & 1975 & 7 & 3 & 18.5 & 79.5 & & & & 3.2 & & UKHYB \\
\hline 31 & 1977 & 9 & 30 & 18.08 & 81.5 & & 3.3 & & 3.3 & & GBA \\
\hline 32 & 1979 & 8 & 29 & 18.24 & 81.3 & & 3 & & 3 & & GBA \\
\hline 33 & 1979 & 4 & 22 & 18.5 & 80.8 & & 3.5 & & 4.7 & & INR \\
\hline 34 & 1981 & 12 & 4 & 18.16 & 81.4 & & 3 & & 3 & & GBA \\
\hline 35 & 1981 & 12 & 16 & 18.57 & 80.7 & & 3.3 & & 3.3 & & GBA \\
\hline 36 & 1983 & 4 & 8 & 18.17 & 81.3 & & 3 & & 3 & & GBA \\
\hline 37 & 1984 & 4 & 24 & 18.27 & 78.8 & & 3.4 & & 3.4 & & GBA \\
\hline 38 & 1984 & 4 & 27 & 18.16 & 79.4 & & 3.4 & & 3.4 & & GBA \\
\hline 39 & 1984 & 6 & 20 & 20.4 & 78.5 & & 3.7 & & 4.3 & & GBA \\
\hline
\end{tabular}


Seismic Hazard Analysis Of Low Seismic Regions, Durg \& Rajnandgaon

\begin{tabular}{|c|c|c|c|c|c|c|c|c|c|c|l|}
\hline 40 & 1985 & 1 & 6 & 20.22 & 78.4 & & 4.2 & & 4.8 & & GBA \\
\hline 41 & 1985 & 9 & 27 & 19.39 & 78.9 & & 3 & & 3 & & GBA \\
\hline 42 & 1986 & 4 & 9 & 18.34 & 82 & & 3.1 & & 3.1 & & GBA \\
\hline 43 & 1987 & 4 & 18 & 22.53 & 79.2 & & & 4.8 & 4.8 & 20 & ISC \\
\hline 44 & 1987 & 4 & 18 & 22.35 & 79.3 & & & 4.9 & 4.9 & 33 & GSPDE, UKHYB \\
\hline 45 & 1990 & 6 & 9 & 18.1 & 80.5 & & 4 & & 4.6 & & CVR \\
\hline 46 & 1996 & 2 & 12 & 22.62 & 82.7 & & & & 4.3 & 33 & MLDMIV, UKHYB \\
\hline 47 & 1997 & 5 & 21 & 23.07 & 80 & & & 6 & 6.7 & 36 & CGS \\
\hline 48 & 1997 & 5 & 21 & 23.08 & 80 & & & 6 & 6.7 & 36 & NEIC, GS \\
\hline 49 & 1997 & 6 & 4 & 23.14 & 80 & & & & 3.9 & 33 & MDHYR, PDE, NEIC \\
\hline 50 & 1998 & 3 & 9 & 22.49 & 78 & & & 4.3 & 4.3 & 10 & GSPDE, NEIC \\
\hline 51 & 2000 & 10 & 10 & 23.8 & 82.7 & & & 4.5 & 4.5 & 33 & GSPDE, NEIC \\
\hline 52 & 2000 & 10 & 16 & 23.28 & 80.3 & & & 4.7 & 4.7 & 33 & GSPDE, NEIC \\
\hline 53 & 2001 & 6 & 12 & 22.22 & 83.9 & & & 4.8 & 4.8 & 33 & GSPDE, NEIC \\
\hline 54 & 2007 & 4 & 13 & 22.70 & 83.2 & & & & 3.1 & 10 & RAIG., IMD \\
\hline 55 & 2011 & 2 & 8 & 22.5 & 79.6 & & & & 3.5 & 12 & SEONI,MP,IMD \\
\hline
\end{tabular}

\section{APPENDIX I1}

Table 2.1Magnitude-Frequency Data of District Headquarters Durg and Rajnandgaon Observation Period- 166 years [1846-2012]

\begin{tabular}{|c|c|c|c|}
\hline S.No. & Moment Magnitude Mw & No. of Earthquake $\geq \mathrm{Mw}$ & No. of Earthquake $\geq \mathrm{Mw}$ per year \\
\hline 1 & 3.0 & 55 & 0.331320 \\
\hline 2 & 3.1 & 48 & 0.289152 \\
\hline 3 & 3.2 & 46 & 0.277104 \\
\hline 4 & 3.3 & 43 & 0.259032 \\
\hline 5 & 3.4 & 41 & 0.246984 \\
\hline 6 & 3.5 & 39 & 0.234936 \\
\hline 7 & 3.6 & 38 & 0.228912 \\
\hline 8 & 3.7 & 38 & 0.228912 \\
\hline 9 & 3.8 & 35 & 0.210840 \\
\hline 10 & 3.9 & 35 & 0.210840 \\
\hline 11 & 4.0 & 34 & 0.204816 \\
\hline 12 & 4.1 & 32 & 0.192768 \\
\hline 13 & 4.2 & 32 & 0.192768 \\
\hline 14 & 4.3 & 32 & 0.192768 \\
\hline 15 & 4.4 & 28 & 0.168672 \\
\hline 16 & 4.5 & 28 & 0.168672 \\
\hline 17 & 4.6 & 26 & 0.156624 \\
\hline 18 & 4.7 & 23 & 0.138552 \\
\hline 19 & 4.8 & 20 & 0.120480 \\
\hline 20 & 4.9 & 14 & 0.084336 \\
\hline 21 & 5.0 & 13 & 0.078312 \\
\hline 22 & 5.1 & 12 & 0.072288 \\
\hline 23 & 5.2 & 12 & 0.072288 \\
\hline 24 & 5.3 & 12 & 0.072288 \\
\hline 25 & 5.4 & 11 & 0.066264 \\
\hline 26 & 5.5 & 11 & 0.066264 \\
\hline 27 & 5.6 & & 0.060240 \\
\hline
\end{tabular}


Seismic Hazard Analysis Of Low Seismic Regions, Durg \& Rajnandgaon

\begin{tabular}{|l|l|l|l|}
\hline 28 & 5.7 & 10 & 0.060240 \\
\hline 29 & 5.8 & 9 & 0.054216 \\
\hline 30 & 5.9 & 7 & 0.042168 \\
\hline 31 & 6.0 & 7 & 0.042168 \\
\hline 32 & 6.1 & 5 & 0.030120 \\
\hline 33 & 6.2 & 5 & 0.030120 \\
\hline 34 & 6.3 & 5 & 0.030120 \\
\hline 35 & 6.4 & 5 & 0.030120 \\
\hline 36 & 6.5 & 5 & 0.030120 \\
\hline 37 & 6.6 & 3 & 0.018072 \\
\hline 38 & 6.7 & 3 & 0.018072 \\
\hline
\end{tabular}

Table 2.2 Earthquake Distribution by Time and Magnitude for District Headquarters

\begin{tabular}{|c|c|c|c|c|c|c|c|c|c|}
\hline \multirow[t]{2}{*}{ Time } & \multirow{2}{*}{$\begin{array}{c}\text { Time } \\
\text { Interval T } \\
\text { in years }\end{array}$} & \multicolumn{4}{|c|}{$\begin{array}{l}\text { No. of Cumulative Earthquakes } \\
\text { occurred in the time interval T }\end{array}$} & \multicolumn{4}{|c|}{$\begin{array}{l}\text { Rate of occurrence of Earthquake /year for } \\
\text { the Magnitude }\end{array}$} \\
\hline & & $\begin{array}{c}3-3.9 \\
\mathrm{M}_{\mathrm{w}}\end{array}$ & $\begin{array}{c}4-4.9 \\
\mathrm{M}_{\mathrm{w}}\end{array}$ & $\begin{array}{c}5-5.9 \\
\mathrm{M}_{\mathrm{w}}\end{array}$ & $\begin{array}{c}6-6.9 \\
\mathrm{M}_{\mathrm{w}}\end{array}$ & $\begin{array}{c}3-3.9 \\
\mathrm{M}_{\mathrm{w}}(\mathrm{N} 1)\end{array}$ & \begin{tabular}{|c|}
$4-4.9$ \\
$\mathrm{M}_{\mathrm{w}}(\mathrm{N} 2)$ \\
\end{tabular} & $\begin{array}{c}5-5.9 \\
\mathrm{M}_{\mathrm{w}}(\mathrm{N} 3) \\
\end{array}$ & $\begin{array}{c}6-6.9 \\
\mathrm{M}_{\mathrm{w}}(\mathrm{N} 4)\end{array}$ \\
\hline 2002-2012 & 10 & 2 & 0 & 0 & 0 & 0.2 & 0 & 0 & 0 \\
\hline 1992-2012 & 20 & 3 & 5 & 0 & 2 & 0.15 & 0.25 & 0 & 0.1 \\
\hline $1982-2012$ & 30 & 8 & 10 & 0 & 2 & 0.2667 & \begin{tabular}{|l|}
0.3333 \\
\end{tabular} & 0 & 0.0667 \\
\hline 1972-2012 & 40 & 17 & 12 & 0 & 2 & 0.425 & 0.3 & 0 & 0.05 \\
\hline $1962-2012$ & 50 & 17 & 16 & 2 & 4 & 0.34 & 0.32 & 0.04 & 0.08 \\
\hline $1952-2012$ & 60 & 17 & 20 & 3 & 4 & 0.2833 & 0.3333 & 0.05 & 0.0667 \\
\hline $1942-2012$ & 70 & 17 & 20 & 3 & 4 & 0.2429 & \begin{tabular}{|l|}
0.2857 \\
\end{tabular} & 0.0429 & 0.0571 \\
\hline $1932-2012$ & 80 & 17 & 20 & 3 & 4 & 0.2125 & 0.25 & 0.0375 & 0.05 \\
\hline $1922-2012$ & 90 & 17 & 20 & 3 & 6 & 0.1889 & \begin{tabular}{|l|}
0.2222 \\
\end{tabular} & 0.0333 & 0.0667 \\
\hline $1912-2012$ & 100 & 17 & 20 & 4 & 6 & 0.17 & 0.2 & 0.04 & 0.06 \\
\hline $1902-2012$ & 110 & 17 & 20 & 5 & 6 & 0.1545 & 0.1818 & 0.0455 & 0.0545 \\
\hline $1892-2012$ & 120 & 17 & 20 & 5 & 6 & 0.1417 & \begin{tabular}{|l|}
0.1667 \\
\end{tabular} & 0.0417 & 0.05 \\
\hline $1882-2012$ & 130 & 17 & 20 & 5 & 6 & 0.1308 & \begin{tabular}{|l|}
0.1538 \\
\end{tabular} & 0.0385 & 0.0462 \\
\hline $1872-2012$ & 140 & 18 & 20 & 6 & 6 & 0.1286 & \begin{tabular}{|l|}
0.1429 \\
\end{tabular} & 0.0429 & 0.0429 \\
\hline $1862-2012$ & 150 & 19 & 20 & 6 & 6 & 0.1267 & \begin{tabular}{|l|}
0.1333 \\
\end{tabular} & 0.04 & 0.04 \\
\hline $1852-2012$ & 160 & 21 & 21 & 6 & 6 & 0.1313 & 0.1313 & 0.0375 & 0.0375 \\
\hline 1846-2012 & 166 & 21 & 21 & 6 & 7 & 0.1265 & \begin{tabular}{|l|}
0.1265 \\
\end{tabular} & 0.0361 & 0.0422 \\
\hline
\end{tabular}

Table 2.3 Rate of Occurrence of Magnitude of District Headquarters Durg and Rajnandgaon

\begin{tabular}{|c|c|c|c|c|c|}
\hline $\begin{array}{c}\text { Time Interval } \\
\text { T in year }\end{array}$ & $\frac{1}{\sqrt{T}}$ & $\frac{\sqrt{ }\left(\mathrm{N}_{1} / \mathrm{T}\right)}{\sqrt{\mathrm{T}}}$ & $\frac{\sqrt{ }\left(\mathrm{N}_{2} / \mathrm{T}\right)}{\sqrt{\mathrm{T}}}$ & $\frac{\sqrt{ }\left(\mathrm{N}_{3} / \mathrm{T}\right)}{\sqrt{\mathrm{T}}}$ & $\frac{\sqrt{ }\left(\mathrm{N}_{4} / \mathrm{T}\right)}{\sqrt{\mathrm{T}}}$ \\
\hline 10 & 0.3162 & 0.1414 & 0.0000 & 0.0000 & 0 \\
\hline 20 & 0.2236 & 0.0866 & 0.1118 & 0.0000 & 0.015811 \\
\hline 30 & 0.1826 & 0.0943 & 0.1054 & 0.0000 & 0.008607 \\
\hline 40 & 0.1581 & 0.1031 & 0.0866 & 0.0000 & 0.00559 \\
\hline 50 & 0.1414 & 0.0825 & 0.0800 & 0.0283 & 0.005657 \\
\hline 60 & 0.1291 & 0.0687 & 0.0745 & 0.0289 & 0.004303 \\
\hline 70 & 0.1195 & 0.0589 & 0.0639 & 0.0247 & 0.003415 \\
\hline 80 & 0.1118 & 0.0515 & 0.0559 & 0.0217 & 0.002795 \\
\hline
\end{tabular}


Seismic Hazard Analysis Of Low Seismic Regions, Durg \& Rajnandgaon

\begin{tabular}{|c|c|c|c|c|c|}
\hline 90 & 0.1054 & 0.0458 & 0.0497 & 0.0192 & 0.002869 \\
\hline 100 & 0.1000 & 0.0412 & 0.0447 & 0.0200 & 0.002449 \\
\hline 110 & 0.0953 & 0.0375 & 0.0407 & 0.0203 & 0.002123 \\
\hline 120 & 0.0913 & 0.0344 & 0.0373 & 0.0186 & 0.001863 \\
\hline 130 & 0.0877 & 0.0317 & 0.0344 & 0.0172 & 0.001653 \\
\hline 140 & 0.0845 & 0.0303 & 0.0319 & 0.0175 & 0.001479 \\
\hline 150 & 0.0816 & 0.0291 & 0.0298 & 0.0163 & 0.001333 \\
\hline 160 & 0.0791 & 0.0286 & 0.0286 & 0.0153 & 0.00121 \\
\hline 166 & 0.0776 & 0.0276 & 0.0276 & 0.0148 & 0.001237 \\
\hline
\end{tabular}

Table 2.4 Faults Considered for Hazard Analysis around the District Headquarter Durg

\begin{tabular}{|c|c|c|c|c|c|c|}
\hline $\begin{array}{c}\text { Fault } \\
\text { No. }\end{array}$ & $\begin{array}{c}\text { Fault length } \\
\text { Li in km }\end{array}$ & $\begin{array}{c}\text { Minimum map } \\
\text { distance to the site } \\
\mathrm{D} \text { in km }\end{array}$ & $\begin{array}{c}\text { Focal depth F } \\
\text { in km }\end{array}$ & $\begin{array}{c}\text { Hypo-central } \\
\text { Distance R in } \\
\mathrm{km}\end{array}$ & $\begin{array}{c}\text { Weightage of } \\
\text { fault } \mathrm{W}_{\mathrm{i}}\end{array}$ & $\begin{array}{c}\text { Maximum } \\
\text { potential } \\
\text { magnitude } \mathrm{M}_{\mathrm{u}}\end{array}$ \\
\hline F1 & 75 & 285.995 & 10 & 286.17 & 0.0302 & 5.3 \\
\hline F2 & 46 & 267.067 & 10 & 267.26 & 0.0185 & 3.6 \\
\hline F3 & 140 & 221.310 & 10 & 221.54 & 0.0563 & 7.2 \\
\hline F4 & 78 & 295.772 & 10 & 295.95 & 0.0314 & 7.0 \\
\hline F5 & 76 & 198.402 & 10 & 198.66 & 0.0306 & 7.0 \\
\hline F6 & 38 & 257.176 & 10 & 257.38 & 0.0153 & 4.4 \\
\hline F7 & 70 & 258.658 & 10 & 258.86 & 0.0282 & 4.5 \\
\hline F8 & 585 & 269.231 & 10 & 269.42 & 0.2352 & 5.3 \\
\hline F9 & 51 & 242.429 & 10 & 242.64 & 0.0205 & 5.2 \\
\hline F10 & 83 & 258.855 & 10 & 259.05 & 0.0334 & 4.4 \\
\hline F11 & 124 & 279.578 & 10 & 279.76 & 0.0499 & 5.3 \\
\hline F12 & 108 & 253.582 & 10 & 253.78 & 0.0435 & 4.8 \\
\hline F13 & 182 & 204.697 & 10 & 204.95 & 0.0732 & 5.3 \\
\hline F14 & 38 & 200.335 & 10 & 200.59 & 0.0153 & 5.2 \\
\hline F15 & 91 & 169.963 & 10 & 170.26 & 0.0366 & 5.2 \\
\hline F16 & 71 & 119.711 & 10 & 120.13 & 0.0286 & 6.3 \\
\hline F17 & 70 & 156.865 & 10 & 157.19 & 0.0282 & 6.3 \\
\hline F18 & 44 & 103.318 & 10 & 103.81 & 0.0177 & 6.3 \\
\hline F19 & 25 & 84.162 & 10 & 84.76 & 0.0101 & 6.3 \\
\hline F20 & 58 & 48.093 & 10 & 49.13 & 0.0234 & 6.3 \\
\hline F21 & 125 & 151.865 & 10 & 152.2 & 0.0503 & 3.5 \\
\hline F22 & 180 & 222.259 & 10 & 222.49 & 0.0724 & 3.5 \\
\hline F23 & 130 & 289.363 & 10 & 289.54 & 0.0523 & 6.5 \\
\hline Total= 2488 & & & & & \\
\hline
\end{tabular}

Table 2.5 Faults Considered for Hazard Analysis around the District Headquarter Rajnandgaon

\begin{tabular}{|c|c|c|c|c|c|c|}
\hline $\begin{array}{c}\text { Fault } \\
\text { No. }\end{array}$ & $\begin{array}{c}\text { Fault } \\
\text { length } \\
\mathrm{Li} \\
\text { i } \mathrm{km}\end{array}$ & $\begin{array}{c}\text { Minimum map } \\
\text { distance to the site D } \\
\text { in km }\end{array}$ & $\begin{array}{c}\text { Focal depth } \\
\mathrm{F} \\
\text { in } \mathrm{km}\end{array}$ & $\begin{array}{c}\text { Hypo-central } \\
\text { Distance R in } \\
\mathrm{km}\end{array}$ & $\begin{array}{c}\text { Weightage of } \\
\text { fault } \mathrm{W}_{\mathrm{i}}\end{array}$ & $\begin{array}{c}\text { Maximum } \\
\text { potential } \\
\text { magnitude } \mathrm{M}_{\mathrm{u}}\end{array}$ \\
\hline F1 & 46 & 290.209 & 10 & 290.39 & 0.0198 & 3.6 \\
\hline F2 & 140 & 235.267 & 10 & 235.48 & 0.06 & 7.2 \\
\hline F3 & 76 & 196.07 & 10 & 196.33 & 0.0326 & 7.0 \\
\hline F4 & 38 & 253.602 & 10 & 253.8 & 0.0163 & 4.4 \\
\hline F5 & 70 & 252.993 & 10 & 253.2 & 0.03 & 4.5 \\
\hline F6 & 585 & 277.013 & 10 & 277.2 & 0.2506 & 5.3 \\
\hline
\end{tabular}


Seismic Hazard Analysis Of Low Seismic Regions, Durg \& Rajnandgaon

\begin{tabular}{|c|c|c|c|c|c|c|}
\hline F7 & 51 & 240.641 & 10 & 240.85 & 0.0219 & 5.2 \\
\hline F8 & 83 & 244.212 & 10 & 244.42 & 0.0356 & 4.4 \\
\hline F9 & 124 & 267.844 & 10 & 268.04 & 0.0532 & 5.3 \\
\hline F10 & 108 & 235.807 & 10 & 236.02 & 0.0463 & 4.8 \\
\hline F11 & 182 & 189.664 & 10 & 189.93 & 0.078 & 5.3 \\
\hline F12 & 38 & 184.814 & 10 & 185.09 & 0.0163 & 5.2 \\
\hline F13 & 91 & 158.214 & 10 & 158.53 & 0.039 & 5.2 \\
\hline F14 & 71 & 117.218 & 10 & 117.65 & 0.0305 & 6.3 \\
\hline F15 & 70 & 134.031 & 10 & 134.41 & 0.03 & 6.3 \\
\hline F16 & 44 & 83.09 & 10 & 83.69 & 0.0189 & 6.3 \\
\hline F17 & 25 & 60.86 & 10 & 61.68 & 0.0108 & 6.3 \\
\hline F18 & 58 & 21.245 & 10 & 23.49 & 0.0249 & 6.3 \\
\hline F19 & 125 & 126.175 & 10 & 126.58 & 0.0536 & 3.5 \\
\hline F20 & 180 & 194.317 & 10 & 194.58 & 0.0771 & 3.5 \\
\hline F21 & 130 & 270.773 & 10 & 270.96 & 0.0557 & 6.5 \\
\hline \multicolumn{2}{|l|}{ Total=2335 } & & & & \\
\hline
\end{tabular}

Table 2.6 PGA for $\mathrm{M}_{100}$ Earthquakes at District Headquarter Durg

\begin{tabular}{|c|c|c|c|c|c|c|}
\hline $\begin{array}{c}\text { Fault } \\
\text { No. }\end{array}$ & $\begin{array}{c}\text { Fault } \\
\text { length } \mathrm{L}_{\mathrm{i}} \\
\text { in km }\end{array}$ & $\begin{array}{c}\text { Minimum map } \\
\text { distance to the } \\
\text { site D in km }\end{array}$ & $\begin{array}{c}\text { Focal } \\
\text { depth F } \\
\text { in km }\end{array}$ & $\begin{array}{c}\text { Hypo central } \\
\text { distance R } \\
\text { in km }\end{array}$ & $\begin{array}{c}100 \text { years } \\
\text { recurrence } \\
\text { M }_{100}\end{array}$ & PGA * of Site \\
\hline F1 & 75 & 285.995 & 10 & 286.17 & 4.26 & 0.00059 \\
\hline F2 & 46 & 267.067 & 10 & 267.26 & 3.42 & 0.00024 \\
\hline F3 & 140 & 221.310 & 10 & 221.54 & 5.10 & 0.00282 \\
\hline F4 & 78 & 295.772 & 10 & 295.95 & 4.60 & 0.00080 \\
\hline F5 & 76 & 198.402 & 10 & 198.66 & 4.50 & 0.00184 \\
\hline F6 & 38 & 257.176 & 10 & 257.38 & 3.70 & 0.00039 \\
\hline F7 & 70 & 258.658 & 10 & 258.86 & 4.00 & 0.00055 \\
\hline F8 & 585 & 269.231 & 10 & 269.42 & 4.15 & 0.00060 \\
\hline F9 & 51 & 242.429 & 10 & 242.64 & 4.10 & 0.00073 \\
\hline F10 & 83 & 258.855 & 10 & 259.05 & 4.00 & 0.00055 \\
\hline F11 & 124 & 279.578 & 10 & 279.76 & 4.61 & 0.00094 \\
\hline F12 & 108 & 253.582 & 10 & 253.78 & 4.30 & 0.00084 \\
\hline F13 & 182 & 204.697 & 10 & 204.95 & 4.73 & 0.00224 \\
\hline F14 & 38 & 200.335 & 10 & 200.59 & 3.88 & 0.00086 \\
\hline F15 & 91 & 169.963 & 10 & 170.26 & 4.43 & 0.00233 \\
\hline F16 & 71 & 119.711 & 10 & 120.13 & 4.48 & 0.00466 \\
\hline F17 & 70 & 156.865 & 10 & 157.19 & 4.48 & 0.00289 \\
\hline F18 & 44 & 103.318 & 10 & 103.81 & 4.10 & 0.00378 \\
\hline F19 & 25 & 84.162 & 10 & 84.76 & 3.66 & 0.00298 \\
\hline F20 & 58 & 48.093 & 10 & 49.13 & 4.30 & 0.01385 \\
\hline F21 & 125 & 151.865 & 10 & 152.2 & 3.44 & 0.00085 \\
\hline F22 & 180 & 222.259 & 10 & 222.49 & 3.45 & 0.00039 \\
\hline F23 & 130 & 289.363 & 10 & 289.54 & 4.80 & 0.00106 \\
\hline
\end{tabular}


Seismic Hazard Analysis Of Low Seismic Regions, Durg \& Rajnandgaon

Table 2.7 PGA for $\mathrm{M}_{100}$ Earthquakes at District Headquarter Rajnandgaon

\begin{tabular}{|c|c|c|c|c|c|c|}
\hline $\begin{array}{c}\text { Fault } \\
\text { No. }\end{array}$ & $\begin{array}{c}\text { Fault } \\
\text { length } \mathrm{L}_{\mathrm{i}} \\
\text { in km }\end{array}$ & $\begin{array}{c}\text { Minimum map } \\
\text { distance to the } \\
\text { site D in km }\end{array}$ & $\begin{array}{c}\text { Focal } \\
\text { depth F } \\
\text { in km }\end{array}$ & $\begin{array}{c}\text { Hypo central } \\
\text { distance R } \\
\text { in km }\end{array}$ & $\begin{array}{c}100 \text { years } \\
\text { recurrence } \\
\mathrm{M}_{100}\end{array}$ & PGA * of Site \\
\hline F1 & 46 & 290.209 & 10 & 290.39 & 3.42 & $0.00020^{*}$ \\
\hline F2 & 140 & 235.267 & 10 & 235.48 & 5.00 & 0.00220 \\
\hline F3 & 76 & 196.070 & 10 & 196.33 & 4.55 & 0.00200 \\
\hline F4 & 38 & 253.602 & 10 & 253.8 & 3.70 & 0.00040 \\
\hline F5 & 70 & 252.993 & 10 & 253.2 & 4.05 & 0.00062 \\
\hline F6 & 585 & 277.013 & 10 & 277.2 & 4.90 & 0.00132 \\
\hline F7 & 51 & 240.641 & 10 & 240.85 & 4.10 & 0.00075 \\
\hline F8 & 83 & 244.212 & 10 & 244.42 & 4.05 & 0.00068 \\
\hline F9 & 124 & 267.844 & 10 & 268.04 & 4.60 & 0.00103 \\
\hline F10 & 108 & 235.807 & 10 & 236.02 & 4.30 & 0.00099 \\
\hline F11 & 182 & 189.664 & 10 & 189.93 & 4.40 & 0.00181 \\
\hline F12 & 38 & 184.814 & 10 & 185.09 & 3.90 & 0.00104 \\
\hline F13 & 91 & 158.214 & 10 & 158.53 & 4.42 & 0.00265 \\
\hline F14 & 71 & 117.218 & 10 & 117.65 & 4.60 & 0.00554 \\
\hline F15 & 70 & 134.031 & 10 & 134.41 & 4.50 & 0.00393 \\
\hline F16 & 44 & 83.090 & 10 & 83.69 & 4.10 & 0.00526 \\
\hline F17 & 25 & 60.86 & 10 & 61.68 & 3.70 & 0.00492 \\
\hline F18 & 58 & 21.245 & 10 & 23.49 & 4.32 & $0.03433^{*}$ \\
\hline F19 & 125 & 126.175 & 10 & 126.58 & 3.45 & 0.00120 \\
\hline F20 & 180 & 194.317 & 10 & 194.58 & 3.48 & 0.00055 \\
\hline F21 & 130 & 270.773 & 10 & 270.96 & 4.92 & 0.00143 \\
\hline & & & & & & \\
\hline
\end{tabular}

